\title{
Pensamento Computacional na Educação Básica: Uma Análise da Relação de Questões de Matemática com as Competências do Pensamento Computacional
}

\author{
Erick John Fidelis Costa ${ }^{1}$, Livia Maria Rodrigues Sampaio Campos ${ }^{1}$, \\ Dalton Dario Serey Guerrero ${ }^{1}$ \\ ${ }^{1}$ Laboratório de Práticas de Software (SPLab) \\ Universidade Federal Campina Grande (UFCG) \\ Paraíba - Brazil \\ erickcosta@copin.ufcg.edu.br, livia@computacao.ufcg.edu.br, \\ dalton@computacao.ufcg.edu.br
}

\begin{abstract}
The computational thinking can be applied in various fields and its main objective is to develop problem-solving skills. Its use is still emerging when considered together the disciplines of basic education. The study described here presents an analysis with the objective of identify the relationship of the computational thinking skills with math questions the 8th and 9th years of basic education. The result of this analysis identified a relationship of questions analyzed with the competencies of PC and the stimulus to solving problems through a joint approach (computational thinking and mathematics) can be considered to improve the performance of students in problem solving.
\end{abstract}

Resumo. O pensamento computacional pode ser aplicado em diversas áreas e seu principal objetivo é desenvolver a capacidade de resolução de problemas. A sua utilização ainda é emergente quando considerada em conjunto as disciplinas do ensino básico. $O$ estudo aqui descrito apresenta uma análise com o objetivo de identificar a relação das competências do pensamento computacional com questões de matemática do $8^{\circ}$ e $9^{\circ}$ anos do ensino básico. $O$ resultado dessa análise identificou uma relação das questões analisadas com as competências do PC e que o estímulo a resolução de problemas através de uma abordagem conjunta (pensamento computacional e matemática) pode ser considerada para melhorar o desempenho dos alunos em resolução de problemas.

\section{Introdução}

Pesquisas na área de ensino de computação são cada vez mais comuns. Os impactos positivos inseridos pela computação dentro das séries iniciais de ensino estão cada vez mais presentes [Fernandes and Castro 2013], [Scaico and de Queiroz 2013], [Silva and de Melo 2013], [Dantas et al. 2013]. Esses impactos são proporcionados quando algumas características da computação são trabalhadas dentro de sala de aula. Essas características agrupadas podem ser denominadas como pensamento computacional (PC). O PC está diretamente relacionado com abstração, decomposição de problemas e estratégias algorítmicas que permitem a organização de soluções usando recursos computacionais.

O PC pode ser considerado um tipo de pensamento analítico. Logo, o propósito desta abordagem é organizar soluções através da divisão de problemas como um todo em 
partes menores. Essas partes menores devem ser fáceis de gerenciar e as soluções propostas devem satisfazer o problema por completo. PC não é uma exclusividade de quem trabalha com computação e pode auxiliar diversas áreas de conhecimento (matemática, leitura, biologia, etc) [Wing 2008].

O CSTA K-12 (Computer Science Standards) defende a implantação de disciplinas de computação dentro do ensino básico com o objetivo de estimular capacidades cognitivas essenciais para o desenvolvimento intelectual do aluno através do PC. A proposta é usar o PC como ferramenta de estímulo e motivação para os estudantes [Tucker 2003]. Os benefícios dessa implantação são cada vez mais significativos e abrangem desde o estímulo ao raciocínio lógico até a capacidade de abstrair e solucionar problemas com muito mais facilidade [Burgett et al. 2015].

O objetivo deste estudo é apresentar o primeiro estágio de uma pesquisa que visa a implantação do PC na educação básica através da matemática. Nesta etapa procuramos identificar a relação das questões de matemática do $8^{\circ}$ e $9^{\circ}$ anos da educação básica disponibilizadas por escolas públicas e particulares do município de Campina Grande Paraíba - Brasil com o PC. Os resultados possibilitaram a observação de características do PC para resolução de problemas que já são estimuladas nas questões das escolas e quais precisam ter maior atenção em uma abordagem conjunta para estimular os alunos. Além de identificar diferentes características em relação as competências do PC identificadas nas questões do PISA e nas questões das escolas.

O presente trabalho, além desta introdução, apresenta na seção 2 a fundamentação teórica. Na seção 3, os trabalhos relacionados. Na seção 4, apresentamos o método de coleta para amostra de questões analisadas, a definição dos critérios da análise e o modelo de classificação. Na seção 5, apresentamos os resultados da análise, e por fim, na seção 6, as conclusões e trabalhos futuros.

\section{Pensamento Computacional}

O PC é a compreensão do poder computacional como instrumento para elevar o poder cognitivo e operacional humano, possibilitando-os enxergar situações de diversas perspectivas, aumentando nossa produtividade, criatividade, inventividade [França et al. 2012]. A capacidade de pensar computacionalmente e raciocinar de maneira lógica é uma prática que fortalece o aprendizado, pois eleva a capacidade de solucionar problemas diversos.

A Sociedade Brasileira de Computação vem incentivando estudos nesta área, pois acredita que ao exemplo de outras disciplinas como Física, Matemática, Química e Biologia, é fundamental a introdução de conceitos de ciência da computação na educação básica. Principalmente como forma de aprimorar o raciocínio computacional das crianças e pelo seu caráter transversal a todas as ciências [Andrade et al. 2013].

O PC possui características que são de grande importância para o desenvolvimento cognitivo do indivíduo [Wing 2006]. Essas características podem ser definidas como: conceituar ao invés de programar; é uma habilidade fundamental e não utilitária; é a maneira na qual pessoas pensam e não computadores; complementa e combina a matemática e a engenharia; gera ideias e não artefatos; e é para todos em qualquer lugar [Gouws et al. 2013].

Barr e Stephenson [Barr and Stephenson 2011] defendem que os conteúdos abor- 
V Congresso Brasileiro de Informática na Educação (CBIE 2016)

Anais dos Workshops do V Congresso Brasileiro de Informática na Educação (CBIE 2016)

dados pelo PC seguindo as características apresentadas anteriormente, despertam no indivíduo capacidades cognitivas que são de extrema importância para a sua formação, por exemplo: abstrair problemas complexos, montar procedimentos para resolução de problemas, raciocínio lógico, resolução de problemas em grupo, etc.

\section{Trabalhos Relacionados}

Barcelos et al. [Barcelos and Silveira 2012] discutem sobre a ciência da computação como ciência básica e as possíveis contribuições em um possível relacionamento com a matemática. A principal contribuição do trabalho é a investigação de possíveis caminhos de pesquisa e como as duas disciplinas podem se beneficiar mutuamente no desenvolvimento conjunto de estratégias de ensino. O estudo indica uma possível relação entre habilidades cognitivas comuns em computação através do PC e a matemática do ensino básico. Essas habilidades podem melhorar o aprendizado de diversos conteúdos, visto que, o desempenho dos países latino-americanos em provas específicas de resolução de problemas apresentam um real problema com o aprendizado de matemática e leitura (PISA, SARESP e SIMCE).

No estudo conduzido por Carvalho et al. [Carvalho et al. 2013] os autores discutem as principais dificuldades na implantação de estratégias pedagógicas voltadas para o PC nas escolas de ensino básico brasileiras. Os autores propõem formas de realizar modificações para que isso seja possível. O estudo mostra pesquisas que estão sendo desenvolvidas muitas vezes financiadas por grandes empresas de TI com o intuito de desenvolver a implantação dos conceitos de PC no ensino básico. Os estudos apontam para um impacto positivo no aprendizado com o uso destas técnicas.

A inserção do PC no ensino ainda é uma tarefa que vem sendo desenvolvida. De acordo com Settle et al. [Settle et al. 2012], para atacar melhor a iniciativa é considerável aplicá-la desde o ensino básico, porém é complicado incorporar algo novo em um currículo já estourado. O autor defende que a modificação do currículo dos cursos de educação para trabalhar com conceitos do PC proporcionará aos novos educadores consciência do que se trata o PC, facilitando a inserção do mesmo em sala de aula. Caso a viabilidade da implantação seja alcançada, muitos alunos serão beneficiados dos estímulos proporcionados.

Mestre et al. [Mestre et al. 2015] discutem a escassez de pesquisas relacionadas ao PC aplicado a matemática. Em seu estudo os autores apresentam uma investigação empírica com o objetivo de avaliar a existência de uma relação entre o PC e a matemática utilizando a amostra de questões de matemática disponibilizadas pelo PISA. Os resultados obtidos apontam para a existência de uma relação entre as competências do PC e as questões analisadas. O estudo apresenta a existência de seis de nove conceitos do PC analisados.

A proposta aqui apresentada segue as mesmas características dos estudos apresentados anteriormente e defende que o PC se trabalhado de forma correta e em paralelo a outras disciplinas pode ser uma ferramenta poderosa e estimulante para o aprendizado. $\mathrm{O}$ objetivo do estudo aqui apresentado é relacionar os conceitos do PC com questões de matemática do ensino básico. Isto utilizando uma metodologia de classificação semelhante a apresentada por Mestre et al. [Mestre et al. 2015]. O estudo aqui apresentado difere principalmente na amostra coletada para análise. 
V Congresso Brasileiro de Informática na Educação (CBIE 2016)

Anais dos Workshops do V Congresso Brasileiro de Informática na Educação (CBIE 2016)

\section{Metodologia}

\subsection{Questão de Pesquisa}

Como apresentado anteriormente o objetivo do estudo aqui descrito é analisar uma amostra de questões de matemática que são trabalhadas do $8^{\circ}$ ao $9^{\circ}$ anos em escolas públicas e particulares quanto a relação com as competências do PC. Para caracterizar melhor nosso objetivo formulamos a seguinte questão de pesquisa:

- P1 - As questões de matemática que estão sendo trabalhadas atualmente em escolas públicas e particulares do $8^{\circ}$ ao $9^{\circ}$ ano em Campina Grande apresentam alguma relação com as competências do PC?

\subsection{Método de Coleta das Questões}

As escolas escolhidas para coleta das questões foram cinco escolas do município de Campina Grande submetidas a prova do PISA em 2012. A escolha dessas escolas aconteceu pela relação que as mesmas tinham com a prova do PISA, visto que elas foram selecionadas pelo exame de acordo com métricas de amostragem que as tornam representativas do total de escolas existentes no município. No total foram duas escolas estaduais, duas escolas privadas e uma escola municipal.

Foram coletadas 200 questões e após selecionada uma amostra aleatória de 100 questões (20 questões por escola). Essa seleção foi realizada para viabilizar a análise individual de cada questão. As 100 questões resultantes foram submetidas a análise de relação com PC, seguindo os critérios e o método de classificação apresentados na sequência deste trabalho.

\subsection{Definição das Competências Analisadas}

No trabalho de Barr e Stephenson [Barr and Stephenson 2011] os autores apresentam competências da computação que se trabalhadas em paralelo a outras disciplinas do ciclo básico de ensino podem estimular a capacidade de resolução de problemas. Os critérios apresentados pelos autores foram utilizados como base para a analise de relação da amostra de questões com o PC. Os critérios da avaliação foram:

- coleta de dados - obter ou gerar dados através de observações empíricas ou de figuras, tabelas, listas, gráficos, etc. Esses dados devem ser usados para resolver o problema proposto;

- análise de dados - interpretar informações a partir de dados fornecidos, onde essas informações serão utilizadas para resolver o problema proposto;

- representação de dados - gerar gráficos, listas, tabelas, matrizes, conjuntos ou diagramas a partir de dados coletados ou analisados;

- problema de decomposição - resolver uma expressão aritmética ou lógica;

- abstração - analisar um contexto real visando obter dados e expressões relevantes para resolução de um problema;

- algoritmos e procedimentos - resolver um problema criando e utilizando uma sequência lógica de passos que não estejam explícitos na questão; 
V Congresso Brasileiro de Informática na Educação (CBIE 2016)

Anais dos Workshops do V Congresso Brasileiro de Informática na Educação (CBIE 2016)

- automação - usar ferramentas para facilitar a resolução de um problema, como: geometer, sketch pad, star logo, python code, snippets e até mesmo uma simples calculadora;

- paralelização - resolver sistemas lineares ou fazer operações com matrizes;

- e simulação - realizar modificações dos valores nas variáveis visando obter e enxergar conclusões ou comportamentos diferentes para o problema proposto.

Foram considerados pesos iguais para qualquer competência e definido um grau de relação para a quantidade de competências total encontradas em cada questão. Esse grau de relação não indica que uma questão obrigatoriamente deverá apresentar todas as competências do PC para que exista. Considerando então o nível de relação entre as competências, podemos observar melhor estas definições na Tabela 1

Tabela 1. Graus de relação para média de critérios identificados.

\begin{tabular}{|l|lll|lll|lll|}
\hline Número Médio de Critérios & 1 & 2 & 3 & 4 & 5 & 6 & 7 & 8 & 9 \\
\hline Graus de Relação & \multicolumn{3}{|c|}{ Baixo } & Moderado & \multicolumn{3}{|c|}{ Alto } \\
\hline
\end{tabular}

\subsection{Método de Classificação}

Após a definição de como as questões seriam medidas foi pensado no método de classificação. Basicamente três pesquisadores da área de PC estudaram cada competência do PC e sua aplicabilidade na matemática. Os estudos relacionados as competências foram realizados em três encontros onde os avaliadores discutiam sobre como cada critério poderia ser observado e como ele era apresentado nas questões exemplo. É interessante ressaltar que as questões exemplo não faziam parte da amostra selecionada para análise. Ao final cada avaliador analisou a presença das competências nas questões da amostra individualmente.

Após os três avaliadores concluírem suas respectivas análises isoladamente, foi realizado por decisão majoritária quais competências estavam presentes nas questões. Isso foi feito decidindo que uma competência estaria presente se pelo menos dois dos avaliadores a tivessem identificado na questão, a mesma decisão foi tomada para o restante das questões analisadas. Ao final, as 100 questões da amostra foram analisadas quanto a presença das competências do PC.

\section{Resultados e Discussões}

\subsection{Análise de Relação com o Pensamento Computacional}

O objetivo da análise apresentada a seguir, é responder a principal pergunta de pesquisa anteriormente apresentada sobre a existência de alguma relação das questões do $8^{\circ}$ e $9^{\circ}$ anos de matemática de escolas públicas e particulares com o PC.

A primeira característica que podemos identificar é que o máximo acumulado de competências encontradas em uma questão foi 3, e o mínimo acumulado de competências encontradas em uma questão foi 0. Para que seja possível identificar essas características de forma mais clara, a seguir, podemos observar um sumário com todas as competências observadas por questão avaliada, essas informações foram organizadas na Tabela 2. 
V Congresso Brasileiro de Informática na Educação (CBIE 2016)

Anais dos Workshops do V Congresso Brasileiro de Informática na Educação (CBIE 2016)

Tabela 2. Quantidade de questões por acumulado de competências.

\begin{tabular}{|l|cccccccccc|}
\hline Acumulado de Competências & 0 & 1 & 2 & 3 & 4 & 5 & 6 & 7 & 8 & 9 \\
\hline Quantidade de Questões & 9 & 56 & 30 & 5 & 0 & 0 & 0 & 0 & 0 & 0 \\
\hline
\end{tabular}

A primeira linha da tabela indica a quantidade de critérios que podem ser encontrados em uma questão analisada da amostra e a segunda indica quantas questões foram analisadas e identificadas com aquela determinada quantidade de competências. Os dados apresentados indicam que as questões da amostra apresentam indícios de alguma relação com as competências do PC e de acordo com a tabela de relação, é baixo.

De acordo com informações apresentadas na Tabela 2, podemos observar também que nenhuma questão apresenta as 9 competências do PC, o mesmo vale para 8, 7, 6, 5 e 4. Isso pode nos indicar que a partir das métricas aqui estabelecidas existem indícios de que não existem questões que apresentem forte e moderada relação com o PC no atual cenário de estudo.

Os dados apresentados anteriormente respondem a questão de pesquisa indicando que as questões, de certa forma, apresentam algum tipo de relação com o PC e estimulam capacidades cognitivas importantes para a formação intelectual do aluno.

\subsection{Análise das Competências do Pensamento Computacional}

A análise apresentada a seguir é uma forma de complementar o estudo, ela nos mostra a quantidade total de ocorrências para as competências em todo quantitativo de questões analisadas. O trabalho foi identificar e definir quais competências estão mais presentes nas questões. Quantificando essas informações para que seja possível entender e caracterizar melhor o estudo, podemos observar a Figura 1.

Quantidade de Identificações por Competência Analisada

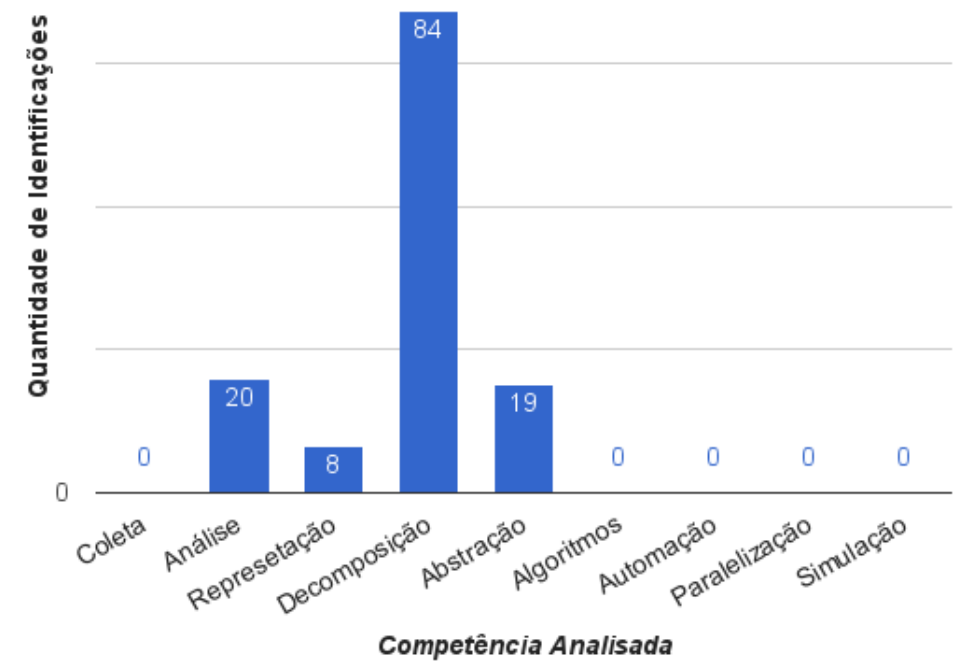

Figura 1. Quantidade acumulada de competências para a amostra analisada.

Analisando a Figura 1 apresentada anteriormente observamos as competências do 
PC e a quantidade acumulada de identificações referentes. Para estes resultados podemos obervar que apenas 5 dos 9 critérios observados foram identificados nas questões da amostra. A competência Problema de Decomposição foi identificado 84 vezes, indicando $64.12 \%$ de ocorrências, sendo assim o critério mais presente nas questões avaliadas. Isto indica que as questões tem relação com a prática e aplicação de ordem em expressões aritméticas.

A Análise de Dados e a Abstração com 20 e 19 identificações representam 15.26\% e $14.5 \%$ das ocorrências, respectivamente. Isto nos mostra que as questões de alguma forma requerem dos alunos que eles interpretem informações a partir de dados fornecidos, além de analisarem contextos reais visando obter dados relevantes para solucionar determinados problemas. A Abstração foi identificada 8 vezes e indica $6.1 \%$ das ocorrências, esse critério é identificado quando a questão de alguma forma solicita a geração de gráficos, listas, tabelas, matrizes ou qualquer outra forma de representação a partir de dados coletados nos problemas apresentados.

Sumarizando as informações para a quantidade de competências identificadas observamos uma média de 1.3 (131 competências identificadas / 100 questões analisadas) competências por questão, indicando uma baixa relação de acordo com as métricas aqui definidas. Essa baixa relação pode ser um fator a ser considerado para tentar explicar o mau rendimento dos alunos em provas que medem a capacidade de resolução de problemas como o PISA e propor estratégias para estimular nos alunos essa capacidade.

Se observamos as características das questões das escolas é possível identificar que elas estão focadas no processo de resolução de expressões algébricas. Ou seja, as questões estão diretamente ligadas a decomposição. Na prática, os alunos estão sendo submetidos a excessivos procedimentos de resolução de cálculos numéricos, deixando de lado capacidades essenciais para o desenvolvimento cognitivo do aluno como a leitura e interpretação de informações relevantes no contexto do problema. No exemplo de questão representado na Figura 2 observamos essa característica.

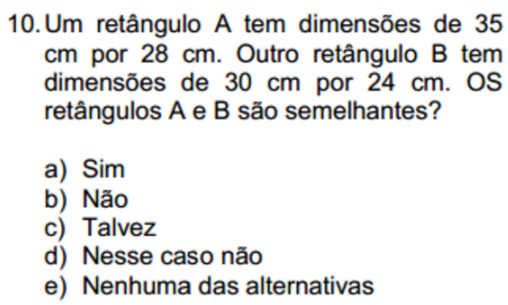

Figura 2. Exemplo de questão das escolas analisada neste estudo

Diferente das questões das escolas aqui analisadas. As questões do PISA apresentam características que estão mais ligadas a análise e interpretação dos problemas para que os mesmos possam ser resolvidos. Isso é caracterizado pela maior identificação das competências Análise de Dados e Abstração como apresentado por Alencar et al. [Mestre et al. 2015]. A Figura 3 mostra um exemplo de questão do PISA analisada no estudo apresentado pelos autores. 
V Congresso Brasileiro de Informática na Educação (CBIE 2016)

Anais dos Workshops do V Congresso Brasileiro de Informática na Educação (CBIE 2016)

\section{FAZENDAS}

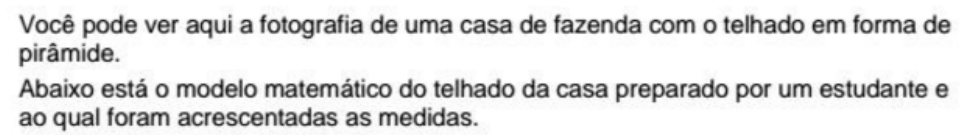

Você pode ver aqui a fotografia de uma casa de fazenda com o telhado em forma de pirâmide.

Abaixo está o modelo matemático do telhado da casa preparado por um estudante e ao qual foram acrescentadas as medidas.

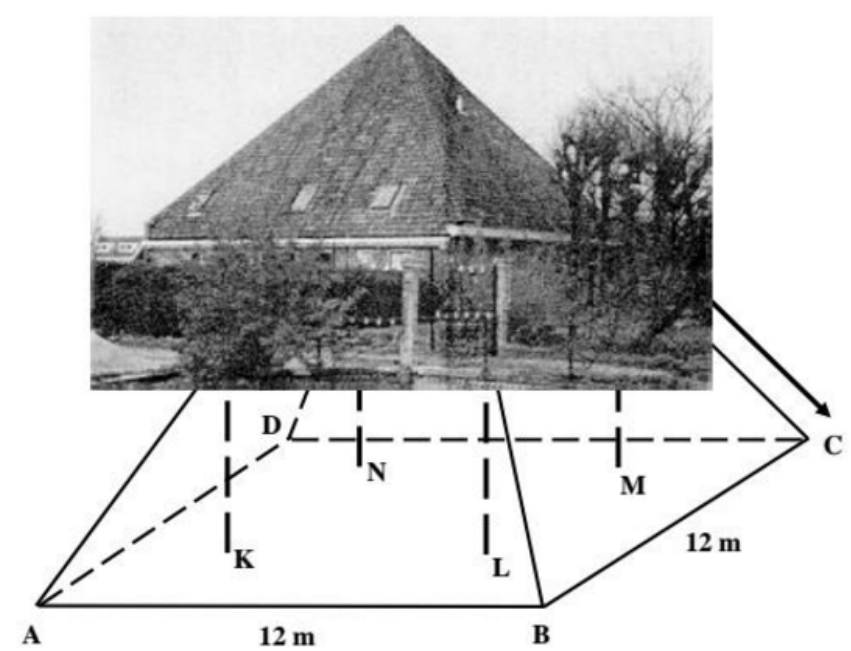

$O$ chão do sótão, denominado $A B C D$ no modelo, é um quadrado. As vigas que suportam o teto são as laterais do bloco (prisma retangular) EFGHKLMN. E está no meio de $\underline{\mathrm{AT}}$, $\mathrm{F}$ está no meio de $\underline{\mathrm{BT}}$, $\mathrm{G}$ está no meio de $\underline{\mathrm{CT}}$ e $\mathrm{H}$ está no meio de $\underline{\mathrm{DT}}$.

Todas as laterais da pirâmide, no modelo, têm o comprimento de $12 \mathrm{~m}$.

Figura 3. Exemplo de questão do PISA analisada no estudo apresentado por Alencar et al. [Mestre et al. 2015]

\subsection{Ameaças a Validade}

Um fator que pode influenciar negativamente são as amostras de questões cedidas pelos professores dessas escolas, de certa forma elas podem ter sofrido algum tipo de seleção destruindo a heterogeneidade dos dados que foram avaliados e ameaçando as conclusões da nossa pesquisa, ou seja, os professores podem submeter apenas suas melhores questões para análise, com isso não saberemos dizer o quão pode ser representativa essa amostra.

Outro fator que deve ser observado é em relação à análise das questões propriamente dita, por limitações de tempo e disponibilidade apenas três avaliadores realizaram a classificação das questões, isso pode ser melhorado e as conclusões podem ser mais específicas se um número maior de avaliadores realizassem o procedimento. Para contornar possíveis impactos negativos, o método de classificação foi conduzido em várias etapas. Como descrito na metodologia deste trabalho.

Existe uma observação em relação as 9 competências utilizados que são bastante utópicos para a formulação de uma questão do ensino básico, acreditamos que pesos iguais podem ser uma boa estratégia para realizar a classificação, mas estudos mais aprofundados podem nos mostrar o verdadeiro impacto de cada competência e os pesos reais para realização de análises mais precisas.

Outra consideração diz respeito aos indícios de uma relação fraca, moderada ou forte das questões com o PC, pois foi um parâmetro definido nesta pesquisa e precisa ser tratado com cuidado. Uma outra visão pode argumentar que uma questão pode estar fortemente relacionada ao PC mesmo que só contemple uma característica. Desta forma, 
V Congresso Brasileiro de Informática na Educação (CBIE 2016)

Anais dos Workshops do V Congresso Brasileiro de Informática na Educação (CBIE 2016)

avanços em pesquisas neste segmente podem fortalecer os conceitos aqui apresentados ou identificar outras maneiras de realizar as análises de relação.

\section{Conclusões}

Tendo em vista que o nosso objetivo era identificar se questões de matemática do $8^{\circ} \mathrm{e}$ $9^{\circ}$ anos de escolas públicas e particulares tem algum tipo de relação com o PC, realizamos uma análise levando em consideração as competências apresentadas na literatura [Barr and Stephenson 2011]. Após analisar os resultados concluímos que:

- As questões de matemática do $8^{\circ}$ e $9^{\circ}$ de escolas públicas e particulares aqui analisadas apresentam algum tipo de relação com o PC.

- Nenhuma parte das questões da amostra tem moderada ou alta relação com o PC. Chegamos a essa conclusão pois nenhuma das questões analisadas foi identificada com pelo menos 4 competências.

- As questões da amostra tem fraca relação com o PC. Chegamos a essa conclusão pois a maior parte das questões analisadas possuía até 3 competências.

- Conseguimos concluir também que a competência que tem maior relação com as questões de matemática da amostra foi a Decomposição seguido de Análise de Dados, Abstração e Representação de Dados, respectivamente.

Como trabalhos futuros pretendemos identificar uma maneira de produzir questões de matemática que contemplem um numero maior de competências do PC e verificar se essas questões podem influenciar o processo de elaboração de soluções para problemas por parte dos alunos. Uma maneira de identificar essa influência é analisando o desempenho dos alunos submetidos a essas novas questões nos testes do PISA focados em matemática. Os resultados desta abordagem poderão validar o método de classificação aplicado neste estudo, pois assim conseguiríamos dizer se questões com maior relação pode influenciar mais os alunos do que questões com uma menor relação.

\section{Referências}

Andrade, D., Carvalho, T., Silveira, J., Cavalheiro, S., Foss, L., Fleischmann, A. M., Aguiar, M., and Reiser, R. (2013). Proposta de atividades para o desenvolvimento do pensamento computacional no ensino fundamental. In Anais do Workshop de Informática na Escola, volume 1, page 169.

Barcelos, T. S. and Silveira, I. F. (2012). Teaching computational thinking in initial series. Proc. CLEI 2012.

Barr, V. and Stephenson, C. (2011). Bringing computational thinking to k-12: what is involved and what is the role of the computer science education community? ACM Inroads, 2(1):48-54.

Burgett, T., Folk, R., Fulton, J., Peel, A., Pontelli, E., and Szczepanski, V. (2015). Dissect: Analysis of pedagogical techniques to integrate computational thinking into k-12 curricula. In Frontiers in Education Conference (FIE), 2015. 32614 2015. IEEE, pages 1-9. IEEE. 
V Congresso Brasileiro de Informática na Educação (CBIE 2016)

Anais dos Workshops do V Congresso Brasileiro de Informática na Educação (CBIE 2016)

Carvalho, T., Andrade, D., Silveira, J., Auler, V., Cavalheiro, S., Aguiar, M., Foss, L., Pernas, A., and Reiser, R. (2013). Discussing the challenges related to deployment of computational thinking in brazilian basic education. In Theoretical Computer Science (WEIT), 2013 2nd Workshop-School on, pages 111-115. IEEE.

Dantas, V. F., de Macedo, E. R., Andrade, J. R. B., Coutinho, D. R. A., Cavalcante, A. F., Vasconcelos, T. G., and Pereira, M. E. d. S. (2013). Combinando desafios e aventura em um jogo para apoiar a aprendizagem de programação em vários níveis cognitivos. In Anais do Simpósio Brasileiro de Informática na Educação, volume 24.

Fernandes, A. M. d. R. and Castro, F. S. (2013). Ambiente de ensino de química orgânica baseado em gamificação. In Anais do Simpósio Brasileiro de Informática na Educação, volume 24 .

França, R. d., Silva, W. d., and Amaral, H. d. (2012). Ensino de ciência da computaçao na educaçao básica: Experiências, desafios e possibilidades. In $X X$ Workshop de Educação em Computação. Curitiba, PR, Brasil.

Gouws, L., Bradshaw, K., and Wentworth, P. (2013). First year student performance in a test for computational thinking. In Proceedings of the South African Institute for Computer Scientists and Information Technologists Conference, pages 271-277. ACM.

Mestre, P., Andrade, W., Guerrero, D., Sampaio, L., da Silva Rodrigues, R., and Costa, E. (2015). Pensamento computacional: Um estudo empírico sobre as questoes de matemática do pisa. In Anais dos Workshops do Congresso Brasileiro de Informática na Educação, volume 4, page 1281.

Scaico, P. D. and de Queiroz, R. J. G. B. (2013). A educação do futuro: uma reflexão sobre aprendizagem na era digital. In Anais do Simpósio Brasileiro de Informática na Educação, volume 24.

Settle, A., Franke, B., Hansen, R., Spaltro, F., Jurisson, C., Rennert-May, C., and Wildeman, B. (2012). Infusing computational thinking into the middle-and high-school curriculum. In Proceedings of the 17th ACM annual conference on Innovation and technology in computer science education, pages 22-27. ACM.

Silva, T. S. and de Melo, J. C. (2013). Cidade dos bits: Um game para auxiliar no aprendizado dos fundamentos da ciência da computação a nível médio. In Anais do Simpósio Brasileiro de Informática na Educação, volume 24.

Tucker, A. (2003). A model curriculum for k-12 computer science: Final report of the acm k-12 task force curriculum committee. Technical report, New York, NY, USA. ACM Order No.: 104043.

Wing, J. M. (2006). Computational thinking. Communications of the ACM, 49(3):33-35.

Wing, J. M. (2008). Computational thinking and thinking about computing. Philosophical transactions of the royal society of London A: mathematical, physical and engineering sciences, 366(1881):3717-3725. 